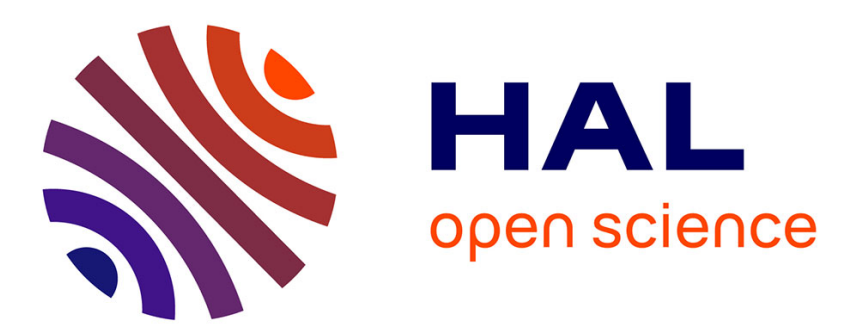

\title{
Comment interpréter la tension eschatologique dans les sources monastiques du haut Moyen Âge ibérique?
}

\author{
Gaelle Bosseman
}

\section{To cite this version:}

Gaelle Bosseman. Comment interpréter la tension eschatologique dans les sources monastiques du haut Moyen Âge ibérique?. Revue Mabillon, revue internationale d'histoire et de littérature religieuses, 2018, pp.27-44. hal-02442537

\section{HAL Id: hal-02442537 \\ https://hal.science/hal-02442537}

Submitted on 21 Jan 2020

HAL is a multi-disciplinary open access archive for the deposit and dissemination of scientific research documents, whether they are published or not. The documents may come from teaching and research institutions in France or abroad, or from public or private research centers.
L'archive ouverte pluridisciplinaire HAL, est destinée au dépôt et à la diffusion de documents scientifiques de niveau recherche, publiés ou non, émanant des établissements d'enseignement et de recherche français ou étrangers, des laboratoires publics ou privés. 


\title{
COMMENT INTERPRÉTER LA TENSION ESCHATOLOGIQUE DANS LES SOURCES MONASTIQUES DU HAUT MOYEN ÂGE IBÉRIQUE? »
}

\author{
par \\ Gaelle BOSSEMAN
}

Juan Gil dans un article majeur de 1978 sur les textes eschatologiques ibériques écrit que la spiritualité rêveuse de la fin $\mathrm{du} \mathrm{Ix}^{\mathrm{e}}$ siècle ", à rapprocher des chimères eschatologiques " qu'il pense partagées par les chrétiens de l'époque, "se reflète elle-même dans l'essor du monachisme " ${ }^{1}$. De même, Adeline Rucquoi en 1999 liait vocation monastique - et même érémitique - et eschatologie affirmant par exemple que « la perspective d'une fin imminente pousse alors hommes et femmes à renoncer au monde et à fuir dans le désert en recherche de la purification et d'une vie entièrement dédiée à la prière et à la pénitence » ${ }^{2}$. Ainsi, si aux viII et Ix $^{\mathrm{e}}$ siècles, «les espérances eschatologiques ont mené une grande partie des chrétiens méridionaux à rechercher la fuite dans les déserts ou le martyre ${ }^{3}$, le lien entre fin des temps imminente et le succès d'un monachisme ascétique et érémitique se distendrait d'après A. Rucquoi au $\mathrm{x}^{\mathrm{e}}$ siècle avec le succès d'une règle "plus "douce" et indulgente ", la règle de saint Benoît ${ }^{4}$, alors même que les chrétiens abandonnent leurs espérances eschatologiques ${ }^{5}$.

Les travaux de J. Gil et d'A. Rucquoi sur l'eschatologie dans la péninsule Ibérique au haut Moyen Âge attirent l'attention sur un phénomène déjà souligné plus généralement par différents auteurs, et notamment Jean Leclercq : le rôle et le poids des préoccupations eschatologiques dans le

1. Juan Gil Fernández, "Judíos y cristianos en Hispania (s. viII y ix) ", Hispania sacra. Revista española de historia eclesiástica, t. 31, 1978-1979, p. 9-88, ici p. 76.

2. Adeline RucquoI, "El fin del milenarismo en la España de los siglos x y XI", dans Milenarismos y milenaristas en la Europa medieval. IX Semana de estudios medievales, Nájera, 3-7 de Agosto 1998, dir. José Ignacio de LA Iglesia Duarte, Logrogne, 1999, p. 281-304, ici p. 282-283 : « La perspectiva de un final inminente empuja entonces a hombres y mujeres a renunciar al mundo y a huir al desierto en busca de la purificación y de una vida enteramente dedicada a la oración y a la penitencia ».

3. Ibid., p. 292.

4. Sur la diffusion de la règle bénédictine dans la péninsule Ibérique, voir Antonio Linage Conde, Los origenes del monacato benedictino en la Peninsula Ibérica, 3 vol., León, 1973 (Fuentes y estudios de historia leonesa, 9-11) ; Florian Gallon, Moines aux extrémités de la terre. Fonctions et représentations du monachisme dans la péninsule Ibérique du haut Moyen $\hat{A g e}\left(V I I I^{e}-X I^{e}\right.$ siècle $), 2$ vol., thèse inédite de doctorat en histoire médiévale sous la direction de Patrick Henriet, soutenue devant l'Université Bordeaux Montaigne, 2014, p. 129-178.

5. A. RucQuor, «El fin del milenarismo », art. cit., p. 302-303. 
développement du monachisme ${ }^{6}$. De fait, les discours sur la fin du monde terrestre et l'avènement de la Jérusalem céleste, en tant qu'ils exhortent à considérer le présent comme un intérim et à anticiper sur les récompenses ultimes, entretiennent la vocation monastique voire martyriale dans les écrits des moines. Plus encore, la vie monastique était comprise comme une anticipation de la vie future sur Terre : la continence des moines, la contemplation de Dieu et la prière faisaient d'eux des images des anges ${ }^{7}$. Pour autant, peut-on considérer que les préoccupations pour la fin des temps dans les discours monastiques ibériques témoignent d'un climat apocalyptique ayant pour conséquence un retrait du monde et la fuite dans le désert?

Si des liens particuliers entre l'eschatologie et un monachisme de spiritualité ascétique existent dans les sources ibériques alto-médiévales ${ }^{8}$, il paraît dès lors intéressant de chercher à préciser ces liens en étudiant le sens et la fonction des références à la fin des temps au sein des textes. Pour ce faire nous considérerons les textes lus et écrits en contexte monastique, dans les communautés méridionales comme septentrionales de la Péninsule, entre la conquête islamique de 711 et la fin du xi ${ }^{\mathrm{e}}$ siècle, moment qui marque un tournant dans l'histoire culturelle et spirituelle des monastères. En effet, sous le règne du roi Alphonse VI de León (†1109), les relations avec Cluny s'accentuent; cette influence s'est notamment traduite par l'arrivée de nombreux moines et manuscrits ultra-pyrénéens et par l'abandon progressif de la liturgie et de l'écriture wisigothiques au profit du rit romain et de la minuscule caroline ${ }^{9}$.

Aussi, en premier lieu, il faudra passer en revue les textes copiés et lus dans les monastères ibériques abordant la thématique eschatologique, certains ayant déjà été bien étudiés. Dans ce cadre, il semble particulièrement nécessaire de prêter attention aux discours sur la fin des temps eux-mêmes et à leur contexte afin de distinguer ce qui ressort d'une approche spirituelle et allégorique d'une compréhension apocalyptique ou millénariste. L'étude de ces textes ainsi que de leur contexte manuscrit nous amènera à proposer quelques pistes sur les fonctions de l'eschatologie pour les moines.

6. Jean Leclerco, L'amour des lettres et le désir de Dieu. Initiation aux auteurs monastiques du Moyen Âge, $\mathrm{l}^{\text {re }}$ éd. Paris, 1957 ; Paris, 1990, p. 9-14 ; ID., « La vocation et la séparation du monde ", dans Aux sources de la spiritualité occidentale, Paris, 1964 (Tradition et spiritualité, 4 ; Lumière de la foi, 21).

7. Sur ce thème voir Kassius Hallinger, «Zur geistigen Welt der Anfänge Klunys », Deutsches Archiv für Erforschung des Mittelalters, t. 10, 1954, p. 417-445, trad. Henri Rochais, « Le climat spirituel des premiers temps de Cluny ", Revue Mabillon, t. 46, 1956, p. 117-140, ici p. 121-130 ; García M. Colombás, Paradis et vie angélique. Le sens eschatologique de la vocation chrétienne, trad. fr. Suitbert CARon, Paris, 1961 (Spiritualité monastique, 1), p. 244-252.

8. Patrick Henriet, Hispania Sacra. Le discours des clercs et la construction d'une identité chrétienne en péninsule Ibérique occidentale (VIII ${ }^{e}$-XII ${ }^{e}$ siècles), mémoire inédit d'Habilitation à diriger des recherches soutenue à l'Université de Versailles-Saint-Quentin, 2003, p. 39-59, ici p. $42-43$; à paraître.

9. Sur l'influence du monachisme clunisien dans la Péninsule et la romanisation de la liturgie, voir Thomas Deswarte, Une chrétienté romaine sans pape. L'Espagne et Rome (586-1085), Paris, 2010 (Bibliothèque d'histoire médiévale, 1), p. 123-172, 403-484. 


\section{Constance de la tension eschatologique dans les sources ibériques ( VIII ${ }^{\mathrm{e}}-\mathrm{IX}{ }^{\mathrm{e}}$ siècles)}

Florian Gallon, dans une thèse récente consacrée au monachisme hispanique, a étudié avec précision la motivation sotériologique de l'entrée au monastère dans les sources nord-péninsulaires. D'après ses observations, l'espoir de rémission des péchés et de salut individuel dans la perspective de la fin du monde et du Jugement est la motivation la plus couramment exprimée dans les actes de donation et les chartes, sans que celle-ci soit propre à la Péninsule ${ }^{10}$. Somme toute relativement traditionnelle, la fonction de l'eschatologie dans ces textes est plus largement à replacer dans les courants de la spiritualité monastique occidentale ${ }^{11}$. La perspective de la fin, la sienne propre ou le Jugement général des âmes, est une préoccupation spirituelle qui fait largement partie des topö̈ des discours monastiques sans qu'il soit nécessaire d'y voir les échos d'un climat apocalyptique. C'est donc plutôt à travers un autre cas largement abordé dans l'historiographie, celui du moine et sans doute abbé Beatus de Liébana, que les indices de ce climat ont été les plus discutés.

Un climat apocalyptique dans les monastères du nord de la Péninsule? Le témoignage de Beatus de Liébana

Beatus est l'auteur présumé d'un commentaire sur l'Apocalypse écrit en 776 et qui a connu un succès considérable dans la Péninsule tout au long du haut Moyen Âge ${ }^{12}$. L'hypothèse que Beatus se serait cru vivre dans l'imminence de la fin des temps prend racine dans un calcul de la date de fin du sixième âge intégré dans le commentaire. Dans la tradition apocalyptique "prédictive " ${ }^{13}$, les computistes attribuent aux six âges du monde hérités

10. F. Gallon, Moines aux extrémités de la terre, op. cit., p. 370-391. L'auteur note que l'imbrication étroite entre vie monastique, ascèse, pénitence et eschatologie est un motif récurrent qui n’est pas propre aux sources péninsulaires, p. 374.

11. Ibid., p. 371 et 374-377. Voir parallèlement le chapitre consacré aux «Significations et motivations du monachisme " dans l'article "Monachisme ", du Dictionnaire de spiritualité ascétique et mystique. Doctrine et histoire [...] Tome X. Mabille-Mythe, dir. Charles BaumGartner et Michel Olphe-Gaillard, Paris, 1959, col. 1547-1558.

12. Devant l'importance de la bibliographie nous renvoyons aux bibliographies des travaux cités. Voir, sur Beatus et le Tractatus, Roger GRYson, "Introduction ", dans Beati Liebanensis tractatus de Apocalipsin, éd. R. Gryson, 2 vol., Turnhout, 2012 (Corpus christianorum. Series latina, 107/B-C), p. XI-CLXVIII ; sur Beatus et les Beatus, Actas del Simposio para el estudio de los códices del «Comentario al Apocalipsis » de Beato de Liébana, 3 vol., Madrid, 1978-1980 ; sur les manuscrits enluminés voir John W. Williams, The Illustrated Beatus. A Corpus of the Illustrations of the Commentary on the Apocalypse, 5 vol., Londres, 1994-2003 et ID., Visions of the End in Medieval Spain. Catalogue of Illustrated Beatus Commentaries on the Apocalypse and Study of the Geneva Beatus, éd. Therese Martin, Amsterdam, 2017 (Late Antique and Early Medieval Iberia). Voir aussi Seis estudios sobre beatos medievales, dir. Maurilio PÉrez González, León, 2010 et, pour une mise au point récente, F. Gallon, Moines aux extrémités de la terre, op. cit., p. 381-384.

13. Bernard McGinn différencie dans l'eschatologie apocalyptique deux courants, un "prédictif " (predictive) qui tente par le biais de calculs ou en reconnaissant une liste de signes spécifiques de prédire la date de la fin, et un courant "non-prédictif " ou d' "imminence psychologique " (non predictive or psychological imminence) qui manifeste l'idée que la fin est connue de Dieu seul et invite le lecteur à penser le présent dans la perspective de la fin à venir. 
d'Augustin une durée d'existence de 6000 ans. Le monde s'achèvera avec le sixième âge, événement prévu d'après différentes traditions pour l'an $500 \mathrm{ou}$ l'an 800 de l'Incarnation ${ }^{14}$. Dans un passage maintes fois cité de son commentaire, Beatus reproduit le calcul de cette date, qu'il situe en l'an 800 (838 de l'ère hispanique) suivant la date de l'Incarnation transmise par la chronique d'Eusèbe-Jérôme en 5200 et largement adoptée en Occident ${ }^{15}$. Écrivant en 776, soit l'année 5976 du monde, Beatus note donc qu'il reste 25 ans avant l'échéance et conclut, en citant la Chronique d'Isidore de Séville, que le temps qu'il reste est insondable pour l'homme. Il mobilise à la suite les citations classiques sur ce thème de la prédiction de l'heure de la fin des Actes des apôtres, 1,7 et de l'Évangile selon Matthieu, 24, $36^{16}$. Les interprétations de ce passage du Livre iv divergent dans l'historiographie. Si certains y voient le témoignage indéniable de l'adventisme de Beatus ${ }^{17}$, d'autres y lisent au contraire la preuve qu'il n'attachait qu'une importance relative à ces calculs ${ }^{18}$.

Il faut replacer le calcul en contexte. Le développement sur les 6000 ans et le sixième âge est d'abord pour Beatus un argument dans une mise au point contestant une lecture littérale d'un septième millénaire. Il s'oppose ainsi à une interprétation millénariste de l'Apocalypse : à la fin du temps que Dieu a prévu pour la Création, il n'y aura pas de septième âge ou de millénaire de règne céleste sur Terre avant le Jugement mais la fin du temps et l'éternité ${ }^{19}$.

Voir B. MaGinn, « The End of the World and the Beginning of Christendom », dans Apocalypse Theory and the Ends of the World, dir. Malcolm BulL, Oxford, 1995 (Wolfson College Lectures), p. 58-89. Louis Bouyer, La vie de S. Antoine. Essai sur la spiritualité du monachisme primitif, Abbaye S. Wandrille, 1950 (Figures monastiques), p. 126-129, avait déjà souligné que cette «perpétuelle imminence » de la fin est au cœur d'un texte comme la Vita Antonii et se trouve au fondement de la spiritualité monastique.

14. Arno Bonst, The Ordering of Time. From the Ancient Computus to the Modern Computer, trad. Andrew Winnard, Cambridge-Oxford, 1993 ; James T. Palmer, « Calculating Time and the End of Time in the Carolingian World, c. 740-820", The English Historical Review, t. 126, $\mathrm{n}^{\circ}$ 523, 2011, p. 1307-1331.

15. J. T. Palmer, "Calculating Time ", art. cit., p. 1310.

16. Beatus de Liébana, Tractatus de Apocalipsin, éd. cit. R. Gryson, vol. in, iv, 5, paragr. 13 , 17, p. 518: Residuum seculi tempus humanae investigationis incertum est. Omnem enim de hac re questionem dominus noster Iesus Christus abstulit dicens...

17. J. Gil Fernández, "Los terrores del ano 800 », Actas del Simposio, op. cit., vol. I, p. 215-247 ; Richard LANDEs, « Lest the Millennium be Fulfilled. Apocalyptic Expectations and the Pattern of Western Chronography, 100-800 CE ", dans The Use and Abuse of Eschatology in the Middle Ages, dir. Werner Verbeke, Daniel Verhelst et Andries Welkenhuysen, Louvain, 1988 (Mediaevalia Lovaniensia. Series 1. Studia, 15), p. 137-211 ; Jean FloRI, L'islam et la fin des temps. L'interprétation prophétique des invasions musulmanes dans la chrétienté médiévale, Paris, 2007 (L'univers historique), p. 148-156.

18. Noureddine Mezoughi, "Beatus et les "Beatus" ", dans El "Beato " de Saint-Sever. Ms. lat. 8878 de la Bibliothèque nationale de Paris, 2 vol., Madrid, 1984, vol. II, p. 19- 30 ; J. Williams, "Purpose and Imagery in the Apocalypse Commentary of Beatus of Liebana ", dans The Apocalypse in the Middle Ages, dir. Richard K. Emmerson et B. McGinn, IthacaLondres, 1992, p. 217-233, ici p. 222-225 ; P. Henriet, Hispania Sacra, op. cit., p. 39-43.

19. Sur le millénarisme voir Jean DANiÉLOU, « La typologie millénariste de la semaine dans le christianisme primitif ", Vigiliae christianae. A Review of Early Christian Life and Language, vol. 2, $\mathrm{n}^{\circ}$ 1, 1948, p. 1-16; Martine DulaEy, « Jérôme, Victorin de Poetovio et le millénarisme », dans Jérôme entre l'Occident et l'Orient. XVI Centenaire du départ de saint Jérôme de Rome et de son installation à Bethléem. Actes du colloque de Chantilly, septembre 1986, dir. Yves-Marie Duval, Paris, 1988 (Collection des études augustiniennes. Série Antiquité, 122) ; 
Poursuivant, le moine de Liébana insiste sur la vacuité et l'orgueil de chercher à calculer la date de la fin :

Et de la même manière qu'une fois le sixième jour achevé, nous ne lisons aucune œuvre du Seigneur mais au contraire qu'il cessa son ouvrage et se reposa, nous croyons de même que le sixième millénaire, qu'il soit fini ou non, est le jour de la résurrection ; quant au jour et à l'heure, comme le dit le Seigneur lui-même "personne ne le sait, ni les anges du ciel, le Père seul " ${ }^{20}$ et chez un autre évangéliste il est ajouté que "ni le Fils» ${ }^{21}$ ne le sait. Il nous faut en vérité comprendre ceci non selon la lettre mais selon l'idée [...]. Ceux qui assurent que la divinité du Christ ignore les choses futures qui sont écrites sur le jour et l'heure de la fin ne se souviennent pas de la personne du Christ parlant dans Isaïe : «Dans mon cœur est le Jour du Jugement » ${ }^{22}$ où il est évident que le Fils savait, mais qu'il ne voulait pas l'indiquer à ses disciples afin que toujours attendant la mort et sans certitude, ils n'aient pas d'espoir dans le siècle. Car sur le jour comme nous lisons que notre seigneur Jésus Christ a resuscité des morts un dimanche, nous espérons qu'à la fin des temps nous ressusciterons un dimanche. Mais parce qu'après être revenu sur ce jour par des calculs de nombreux dimanches se sont produits, dans quel temps, dans quelle année, à quelle heure, quel jour, dans quelle ère, sera la résurrection, nous ne le savons pas ${ }^{23}$.

Beatus mobilise ensuite deux exemples de l'Ancien Testament pour rappeler que Dieu peut raccourcir le temps de la vie des hommes sur Terreque le moine assimile à un temps de pénitence-ou au contraire le prolonger ${ }^{24}$. Il cite ainsi la Genèse lorsque Dieu décide d'effacer l'homme de la Terre par le Déluge sans attendre la fin des 120 ans qu'il leur avait octroyés (Genèse, 6, 3) puis l'exemple du roi Ézéchias : le roi pieux, atteint d'une maladie mortelle, voit sa vie prolongée de 15 ans en récompense de sa prière et de ses bonnes actions passées (Isaïe, 38, 1-5). La conclusion du raisonnement est claire : avec pédagogie, le moine a assorti sa tentative de calcul de nombreuses mises en garde afin de décourager la compréhension littérale qui pourrait en être faite ${ }^{25}$. En ce sens, le passage trouve sa place dans la compréhension

Sumi Shimahara, «Peut-on parler de millénarisme à l'époque carolingienne ? L'apport de quelques sources exégétiques ", Temas medievales, t. 14, 2006, p. 99-138. Comme le souligne cette dernière, ce type d'argument, copié des sources patristiques, ne signifie pas nécessairement que les exégètes étaient réellement confrontés à ce type d'interprétations.

20. Mt, 24, 36 .

21. Mc, 13,32 .

22. Is, 64,3 .

23. Beatus de Liébana, Tractatus de Apocalipsin, éd. cit., vol. II, iv, 5, paragr. 22-25, p. 520-521 : Et sicut sexto die finito nicil legimus dominum operatum nisi cessare ab operibus et requieuisse, ita credimus sexto miliario, utrum finito an non, diem resurrectionis esse; qua die et ora, ut ait ipse dominus nemo scit, neque angeli celorum, nisi pater solus, et in alio euangelista addidit quod neque filius scierit. Nos uero haec non secundum litteram, sed secundum sensum intelligere debemus [...]. Hii adserunt quod Christi diuinitas ignoret futura quae sunt scripta de die et ora nouissima, non recordantes Christi personam in Esayam loquentis : Dies iudicii ex corde meo; unde manifestum est quod filius sciuit, sed discipulis indicare noluit, ut semper de morte suspecti et incerti spem in seculo non haberent. Nam de die legimus quia, sicut dominus noster Iesus Christus die dominico resurrexit a mortuis, ita nos resurrecturos in nouissimo seculo die dominico speramus. Sed quia reuoluente hunc diem in calculo multi dominici dies efficiuntur, quo tempore, quo anno, qua ora, qua die, qua era resurrectio sit nescimus...

24. Ibid., Iv, 5, paragr. 26-27, p. 521-522.

25. Ibid., Iv, 5, p. 519 ; paragr. 31, p. 523. 
allégorique et spirituelle de l'Apocalypse développée par Beatus ${ }^{26}$. L'injonction répétée de se tenir toujours prêt en vue du Jugement montre que le moine de Liébana reste fidèle à l'imminence non-prédictive ou psychologique de Grégoire le Grand, reprise par Isidore de Séville, dont les œuvres ont nourri le Tractatus ${ }^{27}$. Dans l'économie générale du commentaire, la cinquième section du livre IV explique Apocalypse, 7, 4-12 c'est-à-dire la vision des cent quarante-quatre milliers que Beatus interprète, suivant Tyconius, comme symbole de l'Église entière. Le calcul des âges du monde intervient à la suite d'un développement érudit sur la valeur symbolique des nombres et est suivi, comme nous l'avons vu, de plusieurs appels à une lecture spirituelle de cette durée ${ }^{28}$. Pour Beatus, qui manie tout au long du commentaire différents niveaux d'interprétation et concilie la pensée de différents auteurs ${ }^{29}$, imminence prédictive et imminence psychologique ne sont ici qu'apparemment en contradiction : sans être sur le même plan de lecture, elles servent indéniablement la même fonction, l'exhortation au repentir et à la contrition en vue du royaume céleste.

L'adventisme supposé de Beatus trouve néanmoins sa source dans un autre texte : une lettre d'Élipand de Tolède rapporte que lors de la vigile de Pâques Beatus aurait prédit au peuple que la fin du monde était imminente ${ }^{30}$. Comme cela a déjà parfaitement été noté, ce témoignage est à prendre avec précaution puisqu'il s'insère dans le contexte de la querelle adoptianiste et émane d'un adversaire farouche de Beatus ${ }^{31}$. Pour ne donner qu'un aperçu de la virulence de la polémique entre le moine de Liébana et l'archevêque de Tolède on rappellera que tous deux se traitèrent mutuellement de "précurseur de l'Antichrist ", de "pseudoprophète ", voire de "testicule de l'Antichrist ", dans une utilisation classique de l'imagerie apocalyptique dans la polémique antihérétique ${ }^{32}$. Ainsi, sans négliger le témoignage d'Elipand,

26. Voir, avec les travaux de J. Williams et P. Henriet cités supra, Jacques Fontaine, « Fuentes y tradiciones paleocristianas en el método espiritual de Beato ", dans Actas del Simposio, op. cit., vol. I, p. 75-103 ; N. Mezoughi, "Beatus et les "Beatus" ", art. cit.; Aires Augusto Nascimento, "O "Comentário ao Apocalipse" de Beato de Liébana. Entre gramática e escatologia ", Euphrosyne. Revista de filología clássica, t. 28, 2000, p. 129-156.

27. R. Gryson, "Introduction", art. cit., p. cxxxv. Sur cette tradition voir, en plus de B. MaGinn, J. T. Palmer, « Calculating Time», art. cit., p. 1308-1309.

28. Voir encore Beatus de Liébana, Tractatus de Apocalipsin, éd. cit., vol. iI, iv, 5, paragr. 35-36, p. 523-524 ; sur la valeur symbolique voir Th. Deswarte, « La prophétie de 883 dans le royaume d'Oviedo. Attente adventiste ou espoir d'une libération politique ?", Mélanges de sciences religieuses, t. 58, $\mathrm{n}^{\circ} 1,2001$, p. 39-56, ici p. 50-53 ; S. Shimahara, « Peut-on parler de millénarisme à l'époque carolingienne ?», art. cit., p. 55 ; P. HenRIET, «Apocalypse, messianisme et "présentisme". Remarques sur le temps messianique au Moyen Âge et chez quelques penseurs contemporains ", Écrire l'histoire. Histoire, littérature, esthétique, t. 15, 2015, p. 129-137.

29. Sur la méthode exégétique de Beatus, voir Henri DE Lubac, Exégèse médiévale. Les

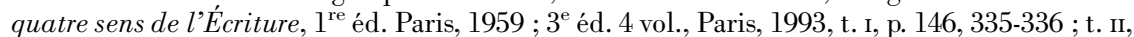
p. 404-405, 475-478 et J. FonTAINe, «Fuentes y tradiciones », art. cit., p. 82-88.

30. ÉLIPAND de Tolède, Epistula episcoporum Hispanie, dans Corpus scriptorum muzarabicorum, éd. Juan Gil Fernández, 2 vol., Madrid, 1973 (Manuales y anejos de « Emerita », 28) (abrégé $C S M$ ), vol. ı, p. 92.

31. J. Williams, «Purpose and Imagery », art. cit. p. 322-323 ; P. Henriet, Hispania Sacra, op. cit., p. 39-40.

32. Élipand de Tolède, Epistula episcoporum Hispanie, éd. cit., vol. I, l. 11, p. 94 ; Epistula ad Albinum, 1. 4-5, p. 96 ; Elipandi epistula ad Felicem nuper conuersum, 1. 9, p. 109 ; BeAtus de LiÉbana et Etherius d'Osma, Adversus Elipandum libri duo, éd. Bengt Löfstedt, Turnhout, 
qui lui-même rapporte les dires d'un tiers, il paraît difficile à interpréter. Le temps précédant Pâques est conçu dans la liturgie comme un temps solennel de préparation ascétique alors que Pâques célèbre la première résurrection qui annonce la résurrection générale des corps après la fin des temps. Beatus concluait son commentaire sur le rappel de la nécessité pour le prédicateur d'exhorter quotidiennement le peuple à la pénitence ${ }^{33}$; dans ce contexte, rien ne contredit l'hypothèse qu'une telle prédiction vise à entretenir le sentiment d'urgence de l' "imminence psychologique » de la fin développé par le Tractatus. L'imminence de la fin est ici avant tout un thème propre à manifester qu'il faut se tenir toujours prêt et vivre dans la crainte du jugement à venir. Qu'en est-il dans les discours des moines méridionaux?

\section{Dans " l'imminence psychologique » de la fin: moines et martyrs au sein des} communautés mozarabes

Comme l'a rappelé récemment Pedro Herrera Roldán, les écrits mozarabes montrent un essor remarquable du monachisme cordouan au $\mathrm{Ix}^{\mathrm{e}}$ siècle ${ }^{34}$. Cet essor, nous l'avons évoqué, a été mis en relation avec un climat apocalyptique, qui serait particulièrement visible dans certains témoignages, en particulier les écrits liés au mouvement des martyrs de Cordoue au milieu du IX ${ }^{\mathrm{e}}$ siècle ${ }^{35}$. D'après Alvare et Euloge de Cordoue, les deux apologétistes du mouvement, cinquante et un chrétiens ont volontairement recherché le martyre en blasphémant publiquement l'islam ou en l'apostasiant entre 851 et 856. Parmi eux, vingt et un étaient moines ${ }^{36}$. Le rôle d'un réseau de monastères cordouans dans le développement du mouvement a été souligné : les futurs martyrs gravitaient autour d'une douzaine d'établissements parmi lesquels Tábanos, centre réputé pour un certain radicalisme et une pratique stricte de l'ascétisme ${ }^{37}$. Ces monastères agissaient à la fois comme lieu de

1984 (Corpus christianorum. Continuatio Mediaevalis, 59), II, chap. 96-99, p. 162-163. Voir Dominique Millet-GÉrard, Chrétiens mozarabes et culture islamique dans l'Espagne des VIII $-I X^{e}$ siècles, Paris, 1984, p. 189-201 ; Florence CLose, Uniformiser la foi pour unifier l'Empire. Contribution à l'histoire de la pensée théologico-politique de Charlemagne, Bruxelles, 2011 (Mémoire de la Classe des Lettres. Collection in- $8^{\circ} .3^{\text {e }}$ série, 59), en particulier p. 43-64 et 83-223.

33. Beatus de LiÉBana, Tractatus de Apocalipsin, éd. cit., vol. II, xiI, chap. 4, paragr. 5, p. 930 ; R. GRYSON, «Introduction », op. cit., p. GXLIV.

34. Pedro Pablo Herrera Roldán, «Sobre monjes y literatura monástica en la Córdoba Emiral ", Meridies, t. 7, 2005, p. 7-28, ici p. 7-8. Sur la situation des communautés chrétiennes d'al-Andalus, voir Cyrille Aillet, Les mozarabes. Christianisme, islamisation et arabisation en péninsule Ibérique (IXe-XII siècle), Madrid, 2010 (Bibliothèque de la Casa de Velázquez, 45), et plus précisément en Bétique, voir p. 69-80.

35. Voir supra et P. P. Herrera Roldán, «Sobre monjes », art. cit., p. 10-11, qui mentionne cette explication parmi d'autres, plus convaincantes.

36. Sur la place des médiations cléricale et monastique dans le mouvement des martyrs, voir P. Henriet, « Sainteté martyriale et communauté de salut. Une lecture du dossier des martyrs de Cordoue (milieu Ix ${ }^{\mathrm{e}}$ siècle) ", dans Guerriers et moines. Conversion et sainteté aristocratiques dans l'Occident médiéval ( $I X^{e}-X I I^{e}$ siècle), dir. Michel Lauwers, Antibes, 2002 (Collection d'études médiévales de Nice, 4), p. 496-567.

37. Jessica A. Coope, The Martyrs of Córdoba. Community and Family Conflict in an Age of Mass Conversion, Lincoln-Londres, 1995, p. 21 ; P. Henriet, « Sainteté martyriale », art. cit., p. 533-537. Voir aussi Kenneth B. Wolf, Christian Martyrs in Muslim Spain, Cambridge, 1988 (Cambridge Iberian and Latin American Studies). 
sociabilité - ils jouaient notamment le rôle de véritables communautés textuelles ${ }^{38}$ - et comme lieu de recrutement des futurs martyrs.

D'après les hypothèses de certains chercheurs, la recherche volontaire du martyre s'expliquerait dans ce contexte par le poids des préoccupations apocalyptiques partagées par les trois communautés religieuses ${ }^{39}$. Cette hypothèse fait de la perspective d'une fin imminente un élément majeur de la vocation monastique et surtout martyriale : persuadés de vivre sous la domination musulmane les tribulations ultimes précédant la fin du monde, ces chrétiens auraient refusé toute compromission avec l'islam et se seraient retirés du monde d'abord par l'entrée au monastère puis par le martyre afin d'assurer leur salut.

Il faut rappeler dans ce contexte que s'il est vrai que les écrits d'Alvare et d'Euloge assimilent Mahomet et l'islam à des précurseurs ou types de l'Antichrist, ces références apocalyptiques ont avant tout une fonction polémique semblable à celle observée dans la lutte contre l'hérésie ${ }^{40}$. Comme l'a bien montré Kenneth B. Wolf, lorsqu'Alvare se livre à l'exégèse de passages bibliques choisis autour de la figure de l'Antichrist, telles que les visions de Daniel 2 et 7, son interprétation reste en tout point conforme à l'interprétation allégorique développée par Jérôme. Son actualisation de l'exégèse pour y intégrer Mahomet et l'islam comme types ou figures de l'Antichrist ne doit pas être comprise comme un indice de sa croyance dans l'imminence de la fin mais bien comme un aspect polémique ${ }^{41}$. De même, lorsque les martyrs Servideus et Roger entrent dans une mosquée et prêchent que la venue du royaume céleste est proche, ce qui annonce la géhenne pour les musulmans qui ne se convertiraient pas au christianisme, Patrick Henriet a souligné que rien n'atteste qu'il faille nécessairement y voir le témoignage d'une conviction apocalyptique. Le propos peut aussi être compris comme une exhortation à la conversion ${ }^{42}$.

Au-delà de cette utilisation polémique de l'imagerie apocalyptique, la préoccupation pour les fins dernières dans les écrits méridionaux reflète

38. Comme l'a souligné P. Henriet, les écrits d'Alvare et d'Euloge laissent entrevoir une circulation dense des textes au sein du groupe des martyrs, P. Henriet, "Sainteté martyriale », art. cit., p. 535-536.

39. J. Gil Fernández, "Judíos y cristianos », art. cit. ; J. Flori, L’islam et la fin des temps, op. cit., p. 160-164. Sur l'hypothèse de l'attente apocalyptique chez Alvare et Euloge, voir Allan H. Cutler, "The Ninth-Century Spanish Martyrs' Movement and the Origins of Western Christian Missions to the Muslims ", The Muslim World, t. 55, n ${ }^{\circ}$ 3, 1965, p. 321-339, ici p. 322-323 ; discussion dans James WaLtz, "The Significance of the Voluntary Martyrs of Ninth-Century Córdoba ", The Muslim World, t. 60, nº 2, 1970, p. 143-159 (n. 28, p. 151) et t. 60, no 3, 1970, p. 226-236, ici, p. 230-232.

40. Paul Alphandéry, "Mahomet-Antichrist dans le Moyen Âge latin ", dans Mélanges Hartwig Derenbourg (1844-1908). Recueil de travaux d'érudition dédiés à la mémoire d'Hartwig Derenbourg par ses amis et ses élèves, Paris, 1909, p. 1-17 ; D. Millet-GÉRARD, Chrétiens mozarabes, op. cit., p. 189-205.

41. K. B. Wolf, "Muhammad as Antichrist in Ninth-Century Córdoba ", dans Christians, Muslims and Jews in Medieval and Early Modern Spain. Interaction and Cultural Exchange, dir. Mark D. Meyerson et Edward D. English, Notre Dame, 1999 (Notre Dame Conferences in Medieval Studies, 8), p. 3-19. Voir aussi l'analyse des procédés polémiques dans les écrits d'Alvare et d'Euloge dans D. Millet-GÉrard, Chrétiens mozarabes, op. cit., p. 108-116.

42. P. Henriet, «Sainteté martyriale», art. cit., n. 134, p. 548. 
également un sentiment d'imminence qu'il faut préciser. On ne trouve que peu de témoignages dans les écrits mozarabes de computs sur l'âge du monde, et ceux-ci ne s'accompagnent pas de tentatives pour calculer la date de la fin mais marquent au contraire un certain scepticisme à cet égard ${ }^{43}$. À l'image des constructions orientales ${ }^{44}$, l'imminence de la fin est plutôt entretenue par la reconnaissance des signes annonçant le retour du Christ ${ }^{45}$. Ainsi, chez Alvare et Euloge, cette tradition eschatologique apocalyptique met l'accent sur la figure de l'Antichrist ou sur la succession des Empires. Pour autant quel sens faut-il accorder au sentiment d'imminence ainsi créé ? Lorsque quelques années avant le mouvement des martyrs, l'ancien maître d'Alvare et d'Euloge, l'abbé Speraindeo du monastère de Saint-Zoüle de Cordoue, est consulté par Alvare au sujet d'un groupe d'hérétiques, il l'encourage à les amener à la pénitence en corrigeant leur erreur : «Car à tout moment méditant notre faute et attendant dans toutes nos prières la fin de notre vie, nous devons faire montre d'une très pieuse compassion envers nos frères au sujet du péché » ${ }^{46}$.

On retrouve chez Speraindeo cette orientation majeure de la spiritualité monastique déjà observée chez Beatus : la nécessité de vivre chaque jour dans l'attente de la consommation du monde et donc de se tenir toujours prêt pour le jour du jugement, ici invoquée pour insister sur la nécessité de ne pas tolérer le mal mais de le corriger. Pour répondre aux hérétiques qui contestent la divinité du Christ en invoquant le fait qu'il ne connaît pas la date de la fin (Évangile selon Matthieu, 24, 36), Speraindeo mobilise le commentaire de Jérôme sur Matthieu qui cite la réponse de Jésus à ses disciples l'interrogeant sur la date, "il ne vous appartient pas de savoir les temps et moments que le Père a fixes de sa seule autorité » (Actes des apôtres, $1,7)$ et commente :

Lorsqu'il dit : «il ne vous appartient pas de savoir», il montre bien qu'il les connaît, lui, mais qu'il n'est pas utile que les apôtres les connaissent afin que, dans l'incertitude continuelle de la venue du Juge, ils vivent chaque jour comme s'ils devaient être jugés le lendemain ${ }^{47}$.

43. Ainsi dans la Chronique mozarabe de 754, le comput final conclut que la rédaction s'achève en 5954 ou en 5950 selon les chiffres utilisés. L'auteur ne dit rien de la fin du sixième âge mais note que cette différence de quatre années n'importe que peu sur une telle somme de temps et cite l'ouvrage à l'interprétation anti-adventiste de Julien de Tolède, le De comprobatione sextae aetatis, éd. Juan Gil Fernández, dans Chronica Hispana saeculi VIII et IX, Turnhout, 2018 (Corpus christianorum. Continuatio mediaevalis, 65), "Chronica Muzarabica », p. 325-382, ici chap. 77, p. 380-382. Alvare, dans une polémique épistolaire avec le juif Bodo-Eléazar, calcule qu'au moment de l'écriture, l'an 840 de l'Incarnation, le monde a 6040 ans, Epistula XVI, dans CSM, éd. cit., vol. I, p. 239. S'appuyant surl'Ancien Testament (Dn, 12,4) Alvare s'opposeà la tentative de calcul d'Eléazar en lui demandant ironiquement comment il pourraît connaître ce que le prophète a scellé, on retrouve ici un argument similaire aux citations de Mt, 24, 36 ou Act, 1, 7 .

44. Sur les traditions apocalyptiques non chronologiques, cf. J. T. PALmER, «Calculating Time ", art. cit., p. 1309.

45. Cf. A. Rucquoi, «El fin del milenarismo », art. cit., p. 285-286.

46. Speraindeo, Epistula, dans CSM, éd. cit., vol. I, p. 203-204 : Unde nostrum omnibus momentis contemplantes delictum et prestolantes uniuersis per oris uite nostre terminum conpassione piissipa pro fratris lapsu [...] deuemus exiuere...

47. D'après l'abbé, il reproduit un extrait d'une controverse islamo-chrétienne qu'il a écrite auparavant, Speraindeo, Epistula, éd. cit., p. 210 = JÉrôme, Commentariorum in Matheum, éd. 
La parenté entre le discours de Speraindeo et celui de Beatus est claire et témoigne d'une véritable continuité quant à la nature de la tension eschatologique. La perspective d'une fin imminente, de fait très présente dans les sources des VIII ${ }^{\mathrm{e}}$-IX ${ }^{\mathrm{e}}$ siècles, met en évidence plutôt qu'un climat imprégné de peurs apocalyptiques, un même héritage, celui de la pensée d'Augustin, de Jérôme et de Grégoire, qui oriente la lecture vers une compréhension spiritualisée de l'imminence de la fin ${ }^{48}$. Cette tradition spirituelle très prégnante dans les discours eschatologiques monastiques méridionaux comme septentrionaux se maintient jusqu'à la fin du xi ${ }^{\mathrm{e}}$ siècle ; on retrouve cet ensemble de citations bibliques sur l'impossibilité de connaître le jour du Jugement et son caractère nécessairement inattendu dans différents textes postérieurs ${ }^{49}$, notamment des sermons ou des homélies ${ }^{50}$. Ceux-ci entretiennent l'auditoire - monastique au vu des manuscrits - dans un état de tension eschatologique permanent ${ }^{51}$.

\section{L'eschatologie en contexte monastique : de l'étude des savoirs à la contemplation des mystères}

La comparaison des textes méridionaux et septentrionaux montre également d'autres points de rapprochement : les références à l'imminence de la

Marc Adriaen et David Hurst, Turnhout, 1969 (Corpus christianorum. Continuatio Mediaevalis, 77), 1. 615-619, p. 232 ; trad. Émile Bonnard, Saint Jérôme. Commentaire sur Matthieu, vol. II, Paris, 1979 (Sources chrétiennes, 259), p. 207.

48. Voir sur la spiritualité eschatologique de Grégoire le Grand et son influence sur la piété médiévale, Claude DAgens, "La fin des temps et l'Église selon saint Grégoire le Grand ", Recherches de science religieuse, t. 58, 1970, p. 273-288.

49. Voir différents exemples de textes hagiographiques, pénitentiels, et liturgiques dans P. Henriet, Hispania Sacra, op. cit., p. $42-43$ et F. Gallon, Moines aux extrémités de la terre, op. cit., p. 390 .

50. Dans la collection des Homélies tolédanes, dont la datation est incertaine (viI ${ }^{\mathrm{e}}$ siècle? postérieure ?), Réginald GrÉGoIRE, Les homéliaires du Moyen Âge. Inventaire et analyse des manuscrits, Rome, 1966 (Rerum ecclesiasticarum documenta. Series maior. Fontes, 6), description et édition partielle de la collection p. 161-185, p. 197-230, voir les sermons $\mathrm{n}^{\text {os }} 62,63,64$, p. 173-174 et 82, 83, 84, p. 177-178; étude de la collection dans Francisco Javier Tovar PAz, Tractatus, sermones atque homiliae. El cultivo del género literario del discurso homilético en la Hispania tardoantigua y visigoda, Cacéres, 1994 (Anuario de estudios filológicos. Anejo, 15), p. 236-274. Voir aussi dans l'homéliaire de Smaragde dont la circulation est bien attestée dans les monastères du nord comme du sud de la Péninsule, une homélie de Bède sur Ac 1, Smaragde DE SaINT-Miniel, Collectiones epistolarum et evangeliorum De tempore et De sanctis, Lectio Actuum apostolorum, I, dans Patrologiae cursus completus [...] Series latina, éd. Jacques-Paul Migne, 221 vol., Paris, 1844-1865 (abrégé PL), vol. cII, col. 309C-310D.

51. Voir les homéliaires de Silos (xi s.) : Londres, British Library, Add. 30.853 (seul témoin complet des Homélies tolédanes), les mss Paris, BnF, NAL 2176 et 2177 qui réunissent les homéliaires de Smaragde et Pierre Diacre mais aussi une homélie de Bède sur le Jugement dernier (Homilia XIV. In dominica tertia post theophaniam, PL, vol. xarv, col. 74D-79A). Le plus ancien homéliaire de Smaragde en Péninsule a été copié à Valeránica, monastère situé près de la frontière islamo-chrétienne, au milieu du $\mathrm{x}^{\mathrm{e}} \mathrm{s}$. (Cordoue, Biblioteca Catedral, Cod. 1, voir Antonio García y García, Francisco Cantelar Rodríguez et Manuel Nieto Cumplido, Catálogo de los manuscritos e incunables de la catedral de Córdoba, Salamanque, 1976 [Bibliotheca salmanticensis. Estudios, 5], p. 3-4) ; un recueil de sermons d'Ephrem le Syrien dont certains abordent le thème du Jugement figurent dans un manuscrit castillan du $\mathrm{x}^{\mathrm{e}} \mathrm{s}$., Paris, BnF, NAL 235, peut-être destiné à une communauté de moniales, voir Manuel C. DíAz y DíAz, Códices visigóticos en la monarquía leonesa, León, 1983 (Colección Fuentes y estudios de historia leonesa, 31), p. 343-344. 
fin du monde sont utilisées dans certains types de discours, en particulier polémiques, réformateurs, ou pénitentiels ; au-delà de ces usages relativement traditionnels et bien étudiés notamment par Bernard McGinn ${ }^{52}$, comment expliquer l'intérêt des moines ibériques pour l'eschatologie? Sans originalité dans l'Occident latin, bibliothèques et scriptoria de la Péninsule lui réservent une place importante. Le commentaire sur l'Apocalypse de Beatus, dont subsistent un peu plus de 35 manuscrits ou fragments, fut un "best-seller monastique " pour la période ${ }^{53}$. Il convient donc à présent de se pencher sur quelques-uns des usages monastiques de ces textes, à travers notamment l'étude de leur contexte manuscrit.

Des indices de lecture et d'étude de la doctrine eschatologique dans les monastères

Les moines s'intéressent en premier lieu à l'eschatologie dans le cadre de l'étude de la Bible et des textes patristiques, ce qu'atteste la présence de traces de lectures ou d'annotations dans les marges de ces textes. Plusieurs manuscrits largement annotés provenant des monastères de San Millán de la Cogolla (Rioja) et de San Sebastián (puis Santo Domingo) de Silos (León) 54 montrent qu'aux $\mathrm{x}^{\mathrm{e}}$ et $\mathrm{xI}^{\mathrm{e}}$ siècles, des textes majeurs sur la doctrine des fins dernières comme les livres XviII à XxiI du De Ciuitate dei d'Augustin ${ }^{55}$, le Prognosticon futuri de Julien de Tolède ${ }^{56}$, les Moralia de Grégoire ${ }^{57}$ ou encore les manuscrits du Tractatus de Beatus ${ }^{58}$, étaient l'objet d'un travail

52. B. McGinn, «The End of the World", art. cit., voir aussi The Encyclopedia of Apocalypticism. Volume 2. Apocalypticism in Western History and Culture, dir. John J. Collins, B. MaGinn et Stephen J. STeIn, New York-Londres, 1998, vol. II.

53. F. Gallon, Moines aux extrémités de la terre, op. cit., p. 384-386.

54. Sur les manuscrits de San Millán et des monastères riojans en général, voir M. C. DíAz y Díaz, Libros y librerías en la Rioja altomedieval, Logrogne, 1991 (Biblioteca de temas riojanos, 28) ; sur les manuscrits de Silos, Ann BoyLan, "The Silos Beatus and the Silos scriptorium", dans Church, State, Vellum, and Stone. Essays on Medieval Spain in Honor of John Williams, dir. Julie A. Harris et Therese Martin, Leide, 2005 (The Medieval and Early Modern Iberian World, 26), p. 173-205; Silos, un milenio. Actas del congreso internacional sobre la Abadia de Santo Domingo de Silos (Burgos y Santo Domingo de Silos, octubre 2001), dir. Blanca Acinas Lope, 4 vol., Burgos, 2003 (Studia silensia, 25).

55. Madrid, Biblioteca de la Real Academia de la Historia (abrégé BRAH), 29 ; Elisa Ruzz García, Catálogo de la sección de Códices de la Real Academia de la Historia, Madrid, 1997, p. 215-217. Édition partielle des notes dans M. C. Díaz y Díaz, « Agustín entre los mozárabes. Un testimonio ", Augustinus. Revista trimestral, t. 25, 1980, p. 157-180.

56. Madrid, Archivo Histórico Nacional, Cod. 1007B ; voir Agustín Millares Carlo, Corpus de códices visigóticos, éd. M. C. DíAz y Díaz, 2 vol., Las Palmas, 1999, nº 127 et Madrid, BRAH, 53 ; E. Ruiz García, Catálogo, op. cit., p. 299-303 ; édition des notes dans Miguel C. Vivancos Gómez, Glosas marginales de los manuscritos visigóticos de San Millán de la Cogolla, Logrogne, 2010 (Aemilianense, colección digital), Apéndice I, p. 227-230. Voir aussi M. C. DíAz y Díaz, Las primeras glosas hispánicas, Barcelone, 1978 (Publicaciones del seminario de literatura medieval y humanística).

57. Santo Domingo de Silos, Ms. 2, éd. M. C. Vivancos Gómez, Glosas y notas marginales de los manuscritos visigóticos del Monasterio de Santo Domingo de Silos, Santo Domingo de Silos, 1996 (Studia silensia, 19), p. 383.

58. Sur le Beatus de l'Escorial, \&.Ir.5 dont l'attribution à San Millán de la Cogolla est incertaine, Guillermo Antolín, Catálogo de los códices latinos de la Biblioteca del Escorial, 4 vol., Madrid, 1910-1916, vol. II., p. 375-376 ; M. C. Vivancos GómEz, Glosas marginales, op. cit., p. 213-214, les notes sont inédites ; sur les notes du Beatus de Silos (Londres, British 
de réflexion d'ordre exégétique ou doctrinal. Discutant le texte, apportant des références scripturaires ou patristiques, ou encore inscrivant en marge le thème discuté afin de faciliter le repérage ou de marquer les étapes du raisonnement, ces annotations étaient vraisemblablement perçues comme solidaires du texte. En effet, dans le cas des Beatus elles forment un corpus que l'on retrouve dans plusieurs manuscrits. Il en est de même pour les deux copies du Prognosticon futuri saeculi de Julien de Tolède dont les gloses et les notes sont identiques ${ }^{59}$. Ces notes érudites ont été copiées avec soin, parfois même mises en valeur dans la mise en page ${ }^{60}$; leur présence n'est pas limitée aux textes eschatologiques mais concerne de manière plus globale les textes doctrinaux et spirituels ${ }^{61}$, ce qui montre bien que la doctrine eschatologique était étudiée par les moines dans le cadre de la lectio diuina ou au même titre que d'autres thématiques.

Les florilèges ou compilations monastiques, qui souvent représentent un moyen d'accès fondamental aux textes pour les moines ${ }^{62}$, démontrent des intérêts similaires. L'excerptatio et la réunion de différents matériaux répondent parfois à des problématiques précises et concrètes (morale, liturgie, polémique) ou servent d'étapes préliminaires à la composition de textes nouveaux ${ }^{63}$. De tels objectifs ont sans doute présidé à la composition de chaînes patristiques mobilisant des textes eschatologiques dans un manuscrit $\mathrm{du} \mathrm{Ix}^{\mathrm{e}}$ siècle originaire d'al-Andalus et arrivé au $\mathrm{x}^{\mathrm{e}}$ siècle dans le monastère de San Cosme y San Damián de Abellar (León) ${ }^{64}$. Un premier ensemble d'extraits patristiques semble plutôt organisé autour d'une orientation

Library, Add. 11695) et des manuscrits de sa famille voir José Antonio Fernández FlóREz, "Beato y los Beatos. El Beato de Escalada y su familia ", dans San Miguel de Escalada (913-2013), dir. Gregoria Cavero Domínguez et Vicente García Lobo, León, 2014 (Folia medievalia, 2), p. 239-272.

59. Une seule note est propre au ms. Madrid, BRAH, 53 (fol. 38v), M. C. Vivancos Gómez, Glosas marginales, op. cit., p. 143 et 227.

60. Voir par exemple les dispositions en cul-de-lampe du Beatus de La Seu d'Urgell, BC 501, fol. 50v, 57, etc. ou les médaillons du Beatus d'Escalada, New York, Morgan Library, Ms. L. 644, fol. 51, 73, de Gérone, BC 1, fol. 74, 92v, 103v, 104, etc. Voir également le cadre stylisé en forme de fleur au fol. 168v.

61. Voir M. C. Vivancos Gómez, Glosas marginales, op. cit., p. 23-24, d'après l'étude des manuscrits glosés et annotés de San Millán et de Santo Domingo de Silos.

62. Ceslas Spico, Esquisse d'une histoire de l'exégèse latine au Moyen Âge, Paris, 1944 (Bibliothèque thomiste, 26), p. 9-26; Silvia Cantelli, Angelomo e la scuola esegetica di Luxeuil, Spoleto, 1990 (Biblioteca di Medioevo latino, 1), p. 13-79 (en particulier p. 60-70) ; Rossana E. GugLielmetTi, «L'editore di esegesi altomedievale tra fonti sommerse e tradizioni creative ", Filologia mediolatina. Rivista della Fondazione Ezio Franceschini, t. 20, 2013, p. 25-68 ; S. Shimahara, « Citations explicites ou recours implicites ? Les usages de l'autorité des Pères dans l'exégèse carolingienne ", dans Les réceptions des Pères de l'Église au Moyen Âge. Le devenir de la tradition ecclésiale. Congrès du Centre Sèvres-Facultés jésuites de Paris (1114 juin 2008), dir. Nicole BÉriou, Rainer Berndt, Michel FÉdou et al., 2 vol., Münster, 2013 (Archa Verbi. Subsidia, 10), vol. I, p. 369-388.

63. Sur le but pratique des compilations, voir J. Leglerco, L'amour des lettres, op. cit., p. 92.

64. Différentes annotations retracent le parcours du manuscrit, León, Archivo Catedralicio, 22, fol. 33v-34, 55v, 15 et 90 ; voir sur ce codex factice composé de deux manuscrits (fol.1-152, 153-156), Fidel FiтA, «El papa Honorio I y san Braulio de Zaragossa », La Ciudad de Dios, t. 4, 1870, p. 189-201, 264-278 ; ID., "Supplemento. Piezas todavía inéditas que contiene el Códice Samuélico ", La Ciudad de Dios, t. 6, 1871, p. 421-429 ; M. C. DíAz y DíAz, Códices visigóticos, op. cit., p. 57-83 ; ID., Manuscritos visigóticos del sur de la Península. Ensayo de distribución regional, Séville, 1995 (Historia y geografía, 11), p. 69-76. 
polémique antijudaïque ${ }^{65}$, une autre chaîne rassemble des extraits d'œuvres diverses d'Augustin, Grégoire ou Isidore principalement autour du livre IV du commentaire In Matheum de Jérôme. On y retrouve le passage commentant l'Évangile selon Matthieu, 24, 36 sur l'imprévisibilité de la date de la fin ; la chaîne s'articule plus largement autour des thèmes du péché depuis Adam jusqu'au présent, et de la résurrection. Annoté et complété à plusieurs reprises, le manuscrit pourrait avoir servi de base pour la rédaction d'un traité ${ }^{66}$; la diversité de son contenu laisse également envisager qu'il ait pu être utilisé comme manuel dans une école monastique ${ }^{67}$.

Ce travail monastique sur les textes eschatologiques n’est pas spécifique à la Péninsule ; ce qui est relativement particulier est plutôt que la composition même du Tractatus de Beatus, véritable compendium de la pensée des Pères sur l'Apocalypse et de manière plus générale sur les fins dernières, a probablement conduit à ce que le commentaire devienne l'un des supports majeurs de ce travail d'étude de l'eschatologie dans les monastères ${ }^{68}$, justifiant le nombre important de copies conservées. Le Tractatus a ainsi été excerpté à différentes époques pour composer des textes courts sur l'Antichrist, sur la date de la fin du monde, ou peut-être encore sur la Jérusalem céleste ${ }^{69}$. Il a également été complété, au $\mathrm{x}^{\mathrm{e}}$ siècle, par de nouveaux textes et images ${ }^{70}$, tandis que certains Beatus ont été augmentés de textes offrant des fonctions propres à ces manuscrits ${ }^{71}$. Ainsi, si l'imminence de la fin est un thème

65. Il s'agit des textes des fol. $11 \mathrm{v}-15$ et $15 \mathrm{v}-17 \mathrm{v}$ qui mobilisent les Étymologies, un commentaire sur Daniel et des textes antijudaïques ; voir F. FITA, « Supplemento », art. cit., et M. C. DíAz y DíAz, Códices visigóticos, op. cit., p. 61-62.

66. La présence dans le manuscrit d'extraits d'un traité polémique sous forme de controverse (fol. $7 \mathrm{v}$-8) qui pourrait être le traité perdu de Speraindeo (cf. supra n. 47), lequel cite certains passages du commentaire de Jérôme sur Matthieu, donne un exemple concret d'une telle possibilité. Voir sur le traité, M. C. DíAz y DíAz, Códices visigóticos, op. cit., p. 78-79.

67. La liste des textes figure dans M. C. DíAz y Díaz, Códices visigóticos, op. cit., p. 61-66. Les plus longs sont le De uiribus illustribus et les épîtres de Braulion de Saragosse, y figurent aussi des poèmes d'Eugène de Tolède, un diagramme sur la Trinité, des extraits des Étymologies sur les plantes ou le De orthographia, des textes géographiques l'Epitaphion Antonine, une Laude Spanie, des extraits de concile, le De genealogiis et enfin le faux décret de Gélase qui liste les écrits canoniques et apocryphes.

68. Travail facilité par le souci pédagogique de Beatus, voir R. GRYson, "Introduction ",

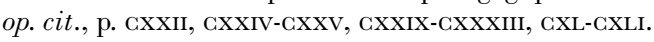

69. Respectivement Indiculus de aduentum Henoch et Eliae, CSM, éd. cit., vol. I, p. 125-140; De fine mundi, dans J. Gil Fernández, "Textos olvidados del Códice de Roda », Habis, t. 2, 1971, p. 165-178, ici p. 170-173 ; l'emprunt est hypothétique pour le dialogue sur la Jérusalem céleste et pourrait s'appuyer plutôt sur la représentation graphique de la cité dans les Beatus, Antoine Hilhorst, "The Escorial Fragment on the Heavenly Jerusalem », dans Media Latinitas. A Collection of Essays to Mark the Occasion of the Retirement of L. J. Engels, dir. Renée I. A. NIP, Hans van DJIK et Elisabeth M. C. Van Houts, Turnhout, 1996 (Instrumenta patristica et mediaevalia, 28), p. 223-228.

70. Sur « la recension posthume » du commentaire voir R. GRYSON, «Introduction », art. cit., p. LI-LXI ; J. Williams, The Illustrated Beatus, op. cit., vol. I, p. 76, 80, 140-141 ; Peter K. KLEIN, "Medieval Apocalypse Cycles and Eschatological Expectations. The So-Called "Terrors" of the Year 1000 ", dans L'Apocalisse nel Medioevo. Atti del convegno internazionale dell'Università degli studi di Milano e della Società internazionale per lo studio del Medioevo latino, Gargnano sul Garda, 18-20 maggio 2009, dir. Rossana E. GuglielmetTi, Florence, 2011 (Millennio medievale, 90 ; Atti di convegni, 27), p. 267-302, ici p. 267-302.

71. Voir par exemple sur le Beatus de Silos, P. Henriet, Hispania Sacra, op. cit., p. 43-45 ; F. Gallon, Moines aux extrémités de la terre, op. cit., p. 385-389. 
central de la réflexion monastique, cet intérêt n'est pas lié à un contexte précis mais reflète plutôt une constante du travail d'étude de la Bible.

Les collections de textes eschatologiques : construction et préservation d'un savoir érudit sur la fin des temps au sein des monastères

Cet intérêt monastique pour les fins dernières se révèle également véritablement érudit voire encyclopédique, dans certaines compilations de savoirs eschatologiques. Dans un manuscrit tel que le Codex de Roda ( $\mathrm{x}^{\mathrm{e}}$ - $\mathrm{xI}^{\mathrm{e}}$ siècles) peut-être composé dans le monastère riojan de San Millán de la Cogolla puis complété et recopié en Navarre, à Santa Maria de Nájera, l'eschatologie est la période ultime d'une histoire de l'humanité construite par compilation de chroniques ${ }^{72}$. Partant d'abord de chroniques universelles (Orose, Isidore), Manuel C. Díaz y Díaz a souligné que le point de vue se centre progressivement sur l'histoire de la Péninsule (les Histoires d'Isidore, les Chroniques d'Alphonse III, d'Albelda puis des listes de rois et comtes pampelonais, aragonais et toulousains) ${ }^{73}$. Enfin, un secteur de textes divers récapitule et prend la mesure de l'ensemble de cette histoire depuis la Création jusqu'à la consommation finale du monde. La dimension érudite des savoirs eschatologiques apparaît par exemple lorsqu'on constate que des extraits de la Chronique du pseudo-Méthode (viI siècle) ont été intégrés dans l'ensemble, non pas pour le récit apocalyptique de la venue du Dernier Empereur, mais afin d'apporter des éléments sur la figure d'Alexandre le Grand et les peuples de Gog et Magog ${ }^{74}$. Un Beatus a également été utilisé pour compléter ce secteur avec des emprunts tant au Tractatus lui-même ${ }^{75}$ qu'aux images ou diagrammes qui accompagnent le commentaire dans les manuscrits ${ }^{76}$.

Le compilateur a ainsi repris de Beatus le résumé de l'histoire du monde dans le cadre des six âges et le calcul des 6000 ans ${ }^{77}$, accompagné des mises

72. Madrid, BRAH, 78, description du codex dans Zacarías García Villada, « El códice de Roda recuperado ", Revista de filologia española, t. 15, 1928, p. 113-130 ; E. RuIz García, Catálogo, op. cit., p. 395-405 ; M. C. DíAz y Díaz, Libros y librerías, op. cit., p. 34-42.

73. Ibid. Voir également Helena de Carlos Villamarín, «El Códice de Roda (Madrid, BRAH 78) como compilación de voluntad historiográfica ", Edad Media. Revista de historia, t. 12, 2011, p. 119-142, et P. Henriet, Hispania Sacra, op. cit., p. 52-53.

74. Édition et étude de la tradition textuelle du texte, Luís Vázouez de PARGa, "Algunas notas sobre el pseudo Metodio y España ", Habis, t. 2, 1971, p. 143-164 ; sur la figure d'Alexandre voir Helena de Carlos Villamarín, « Alejandro en el Códice de Roda », Troianalexandrina, t. 8,2008 , p. $39-58$.

75. Emprunts au liv. iv, chap. 5, fol. 209v ; liv. XI, chap. 6, voir fol. 209 : BEATUs de LiÉBANA, Tractatus de Apocalipsin, éd. cit., vol. II, xI, 6, paragr. 38-39, p. 887 ; fol. 210 : xI, 6, paragr. 4-6, p. $879-880$; paragr. 16, p. 882 ; paragr. 18-19, p. $882-883$; paragr. 33 , p. 886 ; paragr. 38, p. 887.

76. J. Williams, "The Beatus Commentaries and Spanish Bible Illustration", Actas del Simposio, op. cit., vol. II, p. 203-219 ; Yolanta ZALuskA, «Entre texte et image. Les stemmata bibliques au sud et au nord des Pyrénées ", Bulletin de la Société nationale des antiquaires de France, 1986, p. 142-152.

77. Voir supra pour ce calcul chez Beatus. Dans le ms. Madrid, BRAH, 78, fol. 208v, le calcul est une copie de l'Ordo annorum mundi (Recensio quarta breuior), éd. José Carlos MarTínIgLesias, 2 vol., Turnhout, 2014 (Corpus christianorum. Series latina, 115/B-C), vol. II, p. 696-698 ; voir éd. princeps et étude par J. Gil Fernández, "Textos olvidados », art. cit., p. $170-173$. 
en garde du moine de Liébana et d'Augustin ${ }^{78}$. Néanmoins, dans le Codex, le calcul a été mis à jour afin de proposer une date pour la fin des 6000 ans plus proche de la date de l'écriture, donnée en $876^{79}$. La fin du sixième âge est donc annoncée pour l'an 900, soit 25 ans plus tard, sans justification ni révision des chiffres qui aboutissent à ce résultat ${ }^{80}$. Il n'est pas possible de déterminer si le calcul a été mis à jour lors de la copie ou dans le texte qui lui a servi de modèle ${ }^{81}$, néanmoins, la reprise des mises en garde de Beatus tend au moins à attester que le réviseur anonyme souscrivait à son interprétation allégorique ${ }^{82}$. La mise à jour de la date semble quant à elle indiquer que le renouvellement permanent de la tension eschatologique se traduit par le maintien d'une tradition apocalyptique "prédictive" tout autant que "psychologique " dans la construction d'une histoire eschatologiquement orientée.

Des compilations similaires à celles du Codex de Roda, c'est-à-dire insérant les savoirs eschatologiques dans la perspective d'une somme historiographique et géographique universelle, ont été conservées pour les viII et $\mathrm{IX}^{\mathrm{e}}$ siècles ce qui atteste d'une pratique ancienne. Le contenu de ces manuscrits dénote d'un usage peut-être pédagogique ${ }^{83}$; concernant le Codex de Roda, une visée plus directement politique a été postulée ${ }^{84}$. Toutefois, l'orientation encyclopédique paraît mieux à même d'expliquer le sens de la copie de certains textes, on pense par exemple aux prophéties que leur échec a rendues obsolètes ${ }^{85}$, à des textes extra-canoniques ou encore à la réunion de textes aux informations incompatibles ${ }^{86}$. Ainsi, dans le Codex de Roda, un

78. De fine mundi, éd. cit., p. $170=$ Augustin, De ciuitate dei. Libri XI-XXII, éd. Bernard Dombart et Alphonse Kalb, Turnhout, 1955 (Corpus christianorum. Series latina, 48), xviII, 53, p. 652, 1. 12-14: Frustra igitur annos qui remanserunt huic seculo conputare hac definire conamur, quvm « scire nostrum non est " ex ore Ueritatis audiuimus.

79. Ibid., De fine mundi, éd. cit., p. 170-173.

80. Le calcul est faux à plus d'un titre, vraisemblablement en raison de plusieurs erreurs de copistes : comparer Beatus de Liébana, Tractatus de Apocalipsin, éd. cit., p. 571 et De fine mundi, Madrid, BRAH, 78, fol. 208v, p. 170-171.

81. J. GIL FeRnández, «Judíos y cristianos », p. 72-76, signale d'autres manuscrits gravitant dans l'entourage du monastère de San Millán qui présentent des manipulations du calcul jusqu'en 976.

82. P. Henriet, Hispania Sacra, op. cit., p. 42-43.

83. Voir par exemple les mss Albi, Bibl. mun., 29 et Paris, BnF, Latin 484l (Septimanie/Pyrénées ?, viII ${ }^{\mathrm{e}}$ s. et $\mathrm{Ix}^{\mathrm{e}}$ s.) ; El Escorial, Biblioteca del Real Monasterio, R.II.18 (al-Andalus, VIII ${ }^{\mathrm{e}}$ s.-IX ${ }^{\mathrm{e}}$ s.)

84. Sur l'usage politique du discours eschatologique par la monarchie asturo-léonaise, voir supra n. 70 ; Th. Deswarte, «La prophétie de 883 », art. cit.; ID., De la destruction à la restauration. L'idéologie du royaume d'Oviedo-León, VIII $-X I^{e}$ siècles, Turnhout, 2003 (Cultural Encounters in Late Antiquity and the Middle Ages [Printed], 3), p. 147-156.

85. Les textes computistiques ont sans doute été copiés alors que la date était dépassée ; une note marginale, fol. 208v, indique face au comput de l'ordo annorum mundi qui porte la date de 914 : ... est christi ad hinc est usque in era DGGGCLXXIII (973), v̄DGGGLXXV (5875).

86. La coexistence de traditions contradictoires n'est pas exceptionnelle. Au sein même des Beatus, le texte identifie par exemple les deux témoins qui viendront avant l'Antichrist à Élie et Jérémie d'après Victorin (Beatus de LiéBana, Tractatus de Apocalipsin, éd. cit., vol. II, v, ll, p. 639), mais d'autres passages du commentaire (ibid., vol. I, p. 23 et vol. II, p. 674), suivant plutôt les traditions apocryphes, donnent les noms d'Hénoch et d'Élie qui sont ceux figurant dans la plupart des légendes des enluminures, voir J. Williams, The Illustrated Beatus, op. cit., II, p. 71. 
texte évoque un « septième temps " 87 sortant du système augustinien des six âges du monde et suivant peut-être le schéma syriaque du pseudoMéthode ${ }^{88}$; un autre texte sur Adam, la Passion et la Résurrection donne le chiffre de 5227 années entre la Création et l'Incarnation, et non 5199 ou 5099 comme dans les paragraphes précédents ${ }^{89}$. Dans ce manuscrit, la réunion de textes sur la fin des temps indépendamment de leur provenance, de leur concordance ou de leur charge apocalyptique semble dès lors montrer avant tout la volonté de réunir différents matériaux ou traditions dans le but de constituer une somme ouverte à différentes lectures et usages.

\section{Méditation et contemplation sur la fin des temps dans les monastères}

Enfin, un dernier niveau de lecture des textes sur les fins dernières reste à aborder : leur utilisation dans le cadre de la lecture et de la méditation individuelles des moines. C'est probablement à cet effet qu'une apocalypse eschatologique a été insérée au sein de plusieurs homélies dans l'un des secteurs du manuscrit Madrid, Biblioteca de la Real Academia de la Historia, 60, copié puis complété aux $\mathrm{Ix}^{\mathrm{e}}$ et $\mathrm{xI}^{\mathrm{e}}$ siècles dans le nord de la Péninsule ${ }^{90}$. L'Apocalypse se présente comme un dialogue de révélation sur les événements de la fin des temps entre un évêque Alexandre et un roi Aristote ${ }^{91}$. Elle contient principalement une liste de signes décrivant des désordres sociaux ou moraux, puis le récit de la venue de l'Antichrist et sa défaite finale. Le texte a été intégré au sein d'une compilation de sermons qui mêlent un ou deux sermons non identifiés ${ }^{92}$ et des extraits de la collection des Homélies tolédanes ${ }^{93}$. Ces sermons n'étaient vraisemblablement pas destinés à la prédication mais plutôt à la lectio diuina et à la méditation individuelle des

87. Dans le paragraphe intitulé De Antichristo regno, fol. 209 : Item septem tempore ; il s'agit d'une citation modifiée de Beatus de LiéBana, Tractatus de Apocalipsin, éd. cit., vol. iI, xi, 6, paragr. 34-35, p. 886 : item a tempore...

88. Sur ce schéma voir J. T. PALmer, « Calculating Time », art. cit., p. 1326, et sur son insuccès en Péninsule voir J. Gil Fernández, « Judíos y cristianos en la Hispania del siglo vir », Hispania sacra. Revista española de historia eclesiástica, t. 30, 1977, p. 9-110, ici p. 66-67.

89. BRAH, 78, fol. 213v. Cette durée est intéressante car on ne la retrouve que dans la deuxième édition du commentaire de Beatus de LiéBana, Tractatus de Apocalipsin, éd. cit., p. 518. Sur les éditions du commentaire voir R. GRYson, "Introduction ", op. cit., p. Xxxv-LI. Ceci implique que, directement ou non, le compilateur ait eu accès à deux éditions différentes du Tractatus.

90. Il est question ici du secteur C, le codex contenant par ailleurs le Geronticon de Pascase de Dume, la Passio des saints Cosme et Damien et la messe associée, E. Ruiz García, Catálogo, op. cit., p. 327-331 ; édition des textes du manuscrit dans Las Glosas emilianenses, éd. Santos García Larragueta, Logrogne, 1984 (Biblioteca de temas riojanos, 54). Sur l'homéliaire identification des textes dans Adalberto FrAnQUESA, "El códice emilianense 60 y sus piezas litúrgicas ", Hispania Sacra, t. 12, n 24, 1959, p. 423-444.

91. Sur l'interrogatio de nouissimo voir José Guadalajara Medina, El Anticristo en la España medieval, Madrid, 2004 (Arcadia de las letras, 26), p. 44-46.

92. Manuel Ambrosio SÁnchez SÁnchez, "Predicación, gramática y lectura. Glosas a las glosas de San Millán y de Silos ", dans La primitiva predicación hispánica medieval. Tres estudios, Salamanca, 2000 (Publicaciones del SEMYR. Cátalogo de la predicación hispana, 1), ici p. 166-167.

93. Cf. supra, n. 50 . 
moines ${ }^{94}$. La fonction du manuscrit n'a pas été bien cernée; le fait qu'il présente de très nombreuses gloses a été interprété comme l'indice qu'il s'agirait d'un manuel scolaire mais comme l'a noté Manuel Sánchez Sánchez, les gloses ont été portées avant tout sur des textes ascétiques et spirituels, notamment l'Interrogatio de nouissimo, dont l'intérêt pour l'apprentissage du latin est limité. Il faut plutôt, selon son hypothèse, voir dans ces gloses la volonté de mieux comprendre ces textes; et sans doute leur lecture répétée dans le cadre de la méditation individuelle.

Ce rôle est également assuré par la lecture des textes exégétiques et notamment du commentaire sur l'Apocalypse de Beatus. Il revient à Jacques Fontaine d'avoir mis en valeur la fonction de lecture méditative et de support à la contemplation mystique des Écritures des Beatus au sein des monastères ${ }^{95}$. "Outil devenu standard dans l'exégèse monastique au VIII ${ }^{e}$ siècle " ${ }^{96}$, l'interprétation ecclésiologique dominante des commentaires latins de l'Apocalypse au haut Moyen Âge place dans le présent de l'Église la réalisation de l'eschatologie. Ainsi, la compréhension allégorique de l'Apocalypse développée par Beatus s'accompagne d'une actualisation constante mettant en lumière comment les visions prophétiques s'incarnent perpétuellement dans le présent car, comme le soulignait J. Fontaine, le but de Beatus n'est pas d'offrir à ces lecteurs une interprétation «abstraite » ou « essentialiste et platonicienne ». Ces derniers, amenés par la progression des interprétations du texte à reconnaître dans une seule vision l'ensemble des temps passés, présents et futurs, contemplent l'histoire de l'humanité et du schéma divin. Les images, en juxtaposant des éléments illustrant littéralement et allégoriquement le texte, participent de la fonction mystagogique et méditative du commentaire ${ }^{97}$. Ainsi, d'après les mots de J. Fontaine, Beatus invite à « contempler et à accomplir maintenant l'histoire du Salut de telle manière qu'elle se réalise dans ce monde, et surtout dans l'Église, hodie et nunc ", en ce sens «le commentaire a pu apparaître comme un bréviaire spirituel permanent de la vie monastique comme vie monastique et témoignage eschatologique toujours actuel $"{ }^{98}$.

94. M. A. SÁnchez SÁnchez, "Predicación, gramática y lectura ", art. cit., p. 184-186. L'auteur note que dans leur état actuel les textes n'ont pas pu être préchés.

95. J. Fontaine, «Fuentes y tradiciones », art. cit., p. 88-98 ; voir aussi Otto Karl WerckmeIsTER, "The First Romanesque Beatus Manuscripts and the Liturgy of Death", Actas del simposio, op. cit., vol. II, p. 167-182; sur le rôle des Beatus dans la spiritualité ascétique monastique, Th. Deswarte, « La prophétie de 883 », art. cit., p. 53-56 ; ID., De la destruction à la restauration, op. cit, p. 284-289.

96. E. Ann Matrer, "Exegesis of the Apocalypse in the Early Middle Ages », dans The Year 1000. Religious and Social Response to The Turning of The First Millennium, dir. Michael Frassetto, New York-Basingstoke, 2002, p. 29-40, ici p. 34.

97. Voir sur la fonction spirituelle des images dans les Beatus, J. Williams, «Purpose and Imagery ", art. cit. ; Mireille MenTRÉ, La peinture mozarabe. Un art chrétien hispanique autour de l'an 1000, Paris, 1995. Sur l'iconographie des Beatus, voir J. Williams, The Illustrated Beatus, op. cit.; sur la mappa mundi, Patrick Gautier Dalché, " De la glose à la contemplation. Place et fonction de la carte dans les manuscrits du haut Moyen Âge ", dans Testo e immagine nel alto medioevo. [XLI] Settimana di studio del Centro italiano di studi sull'alto medioevo, 2 vol., Spolète, 1994, vol. II, p. 693-764, ici p. 752-757.

98. J. Fontaine, «Fuentes y tradiciones ", art. cit. p. 99 : »... contemplar y cumplir ahora la historia de la Salvación, tal y como se está realizando en este mundo y sobre todo en la Iglesia, hodie et nunc »; ibid., p. 101 : « breviario espiritual permanente de la vida monástica como vida 
L'importance de la tension eschatologique pour les moines ibériques avait déjà été soulignée de longue date par différents chercheurs. L'examen proposé ici de quelques références à la fin du monde dans les textes monastiques a permis de mettre en évidence une constante. Ces références ne doivent vraisemblablement pas être comprises en contexte monastique comme les témoignages d'un climat apocalyptique tellement est prégnante la tradition de l'« imminence psychologique », héritée de l'eschatologie augustinienne et grégorienne. Cette tradition a notamment été véhiculée dans la péninsule Ibérique par le commentaire sur l'Apocalypse de Beatus de Liébana, lequel semble s'être efforcé de concilier eschatologie prédictive et interprétation spiritualisée de l'imminence de la fin.

Si intérêt pour la fin des temps et conviction d'en vivre les derniers moments ne doivent pas être confondus, il n'est pas pour autant aisé de déterminer les fonctions de l'eschatologie apocalyptique pour les moines. Comme l'ont bien montré les travaux de B. McGinn puis de James T. Palmer, dans la tradition grégorienne, le sentiment de l'imminence nourrit des discours pénitentiels et réformateurs. Le survol, assurément trop rapide, de quelques manuscrits ibériques réalisé ici a permis de considérer d'autres contextes, notamment érudits ou contemplatifs, qui n'ont pas été jusqu'à présent l'objet de recherches systématiques. Un examen plus approfondi des textes et de leur contexte manuscrit devra préciser les pistes ébauchées dans le cadre de cette contribution ; néanmoins, nous espérons avoir montré que la diversité même des usages envisagés aura permis de mettre en lumière l'intérêt de réévaluer la tension eschatologique dans les écrits monastiques à l'aune des pratiques culturelles et spirituelles des moines.

\section{Gaelle Bosseman}

EPHE, Université PSL, SAPRAT (EA 4116) 